\title{
Transcriptome analysis of Deinagkistrodon acutus venomous gland focusing on cellular structure and functional aspects using
} expressed sequence tags

\author{
Bing Zhang ${ }^{\dagger 1,2}$, Qinghua Liu ${ }^{\dagger 3}$, Wei Yin ${ }^{4}$, Xiaowei Zhang2, Yijun Huang, \\ Yingfeng $\mathrm{Luo}^{1,2}$, Pengxin $\mathrm{Qiu}^{3}$, Xingwen $\mathrm{Su}^{3}$, Jun $\mathrm{Yu}^{1,2}$, Songnian $\mathrm{Hu}^{1,2}$ and \\ Guangmei Yan*3
}

\begin{abstract}
Address: ${ }^{1}$ James D. Watson Institute of Genome Sciences, Zhejiang University, Hangzhou, China, ${ }^{2}$ Beijing Institute of Genomics, Chinese Academy of Sciences, Beijing, China, ${ }^{3}$ Department of Pharmacology, Zhongshan Medical School of Sun Yat-sen University, Guangzhou, China and ${ }^{4}$ Department of Biochemistry, Zhongshan Medical School of Sun Yat-sen University, Guangzhou, China

Email: Bing Zhang - zhangbin@genomics.org.cn; Qinghua Liu - liuqinghua0918@tom.com; Wei Yin - yinwei@msn.com; Xiaowei Zhang - zhangxw@genomics.org.cn; Yijun Huang - pharmaco@gzsums.edu.cn; Yingfeng Luo - luoyf@genomics.com.cn; Pengxin Qiu - qiupengxin@21cn.com; Xingwen Su - suxw@gzsums.edu.cn; Jun Yu - junyu@genomics.org.cn; Songnian Hu - husn@genomics.org.cn; Guangmei Yan* - guangmeiyan020@yahoo.com.cn

* Corresponding author †Equal contributors
\end{abstract}

Published: 15 June 2006

BMC Genomics 2006, 7:152 doi:10.1186/147|-2164-7-152
Received: 06 May 2006

Accepted: 15 June 2006

This article is available from: http://www.biomedcentral.com/I47/-2 I64/7/I52

(C) 2006 Zhang et al; licensee BioMed Central Ltd.

This is an Open Access article distributed under the terms of the Creative Commons Attribution License (http://creativecommons.org/licenses/by/2.0), which permits unrestricted use, distribution, and reproduction in any medium, provided the original work is properly cited.

\begin{abstract}
Background: The snake venom gland is a specialized organ, which synthesizes and secretes the complex and abundant toxin proteins. Though gene expression in the snake venom gland has been extensively studied, the focus has been on the components of the venom. As far as the molecular mechanism of toxin secretion and metabolism is concerned, we still knew a little. Therefore, a fundamental question being arisen is what genes are expressed in the snake venom glands besides many toxin components?
\end{abstract}

Results: To examine extensively the transcripts expressed in the venom gland of Deinagkistrodon acutus and unveil the potential of its products on cellular structure and functional aspects, we generated 8696 expressed sequence tags (ESTs) from a nonnormalized cDNA library. All ESTs were clustered into 3416 clusters, of which $40.16 \%$ of total ESTs belong to recognized toxincoding sequences; $39.85 \%$ are similar to cellular transcripts; and $20.00 \%$ have no significant similarity to any known sequences. By analyzing cellular functional transcripts, we found high expression of some venom related genes and gland-specific genes, such as calglandulin EF-hand protein gene and protein disulfide isomerase gene. The transcripts of creatine kinase and NADH dehydrogenase were also identified at high level. Moreover, abundant cellular structural proteins similar to mammalian muscle tissues were also identified. The phylogenetic analysis of two snake venom toxin families of group III metalloproteinase and serine protease in suborder Colubroidea showed an early single recruitment event in the viperids evolutionary process.

Conclusion: Gene cataloguing and profiling of the venom gland of Deinagkistrodon acutus is an essential requisite to provide molecular reagents for functional genomic studies needed for elucidating mechanisms of action of toxins and surveying physiological events taking place in the very specialized secretory tissue. So this study provides a first global view of the genetic programs for the venom gland of Deinagkistrodon acutus described so far and an insight into molecular mechanism of toxin secreting.

All sequences data reported in this paper have been submitted into the public database [GenBank: DV5565II-DV565206]. 


\section{Background}

Venomous snakes possess one of the most sophisticated integrated weapons system in the natural world. It has been hypothesized that the snake venom gland itself evolved in the mouth region as a consequence of an evolutionary change in the pancreatic trait, and consequently, some of the toxins should show strong affinities to pancreatic proteins [1]. Recent studies suggested that snake venom components such as phospholipase A2 have evolved in an accelerated manner to acquire their diverse physiological activities [2]. Most studies of snake venom glands have focused almost exclusively on the components of venom. Therefore, a fundamental question being arisen is what genes are expressed in the snake venom glands besides many toxin components? To obtain a more comprehensive view of function of the venom gland, it is necessary for us to choose a proper model to study gene expression and toxin diversity in snake venom gland and perform comparative research with other species.

Deinagkistrodon acutus (D. acutus) is a specific snake in Southern China and many toxin components from the venom gland of $D$. acutus have been purified and characterized [3-6]. More studies for D. acutus venom gland will promote the application of toxin in medicine. To our best knowledge, there is little information about the D. acutus venom gland at the molecular level. This problem is compounded by the limited number of annotated $D$. acutus nucleotide sequences currently deposited in the public databases $(<82)$. For this reason, D. acutus should be a good model for use in studying gene expression and organogenesis in snake venom gland. Analysis of expressed sequence tags (ESTs) is an efficient approach for gene discovery, expression profiling [7-9] and development of resources useful for functional genomics studies. For this purpose, we decided to adopt the strategy of large scale sequencing by constructing a cDNA library from the venom gland of D. acutus. Thus we should be able to find relevant genes and investigate their function after screening and expressing the target genes.

Knowledge of genes and proteins expressed in the various venom glands offers a potential resource to guide future investigations relevant to biology of venom gland and toxicology. For instance, proteins secreted from the venom gland of $D$. acutus represent a subset of candidates potentially involved in local and systemic effects as pain, edema, bleeding and muscle necrosis. Moreover, gene cataloguing and profiling of the venom gland of D. acutus is an essential requisite to provide molecular reagents for functional genomic studies needed for the elucidation mechanisms of action of toxins and the discovery of their antagonists [10]. On the other hand, it will allow the identification of cellular functional transcripts that may represent a general panorama of the physiological events of the venom glands, surveying gene expression from the very specialized secretory tissue.

\section{Results and discussion}

\section{Overview of ESTs from the venom gland of D. acutus}

After discarding the poor-quality sequences, 8696 highquality ESTs were used to analyze gene expression profile in the venom gland of $D$. acutus. The mean read length of ESTs was 398 bp (ranging from 50 bp to 772 bp, Figure 1). Subsequently, ESTs were clustered into 3416 clusters, of which 118 clusters (40.16\% ESTs) associated with toxin function has been reported elsewhere [11]. In this report, we discussed mainly 1184 clusters (39.85\% ESTs) involved in the cellular functional transcripts and other novel sequences (Figure 2). The distribution of all ESTs was followed:

(1) Twenty-five clusters consisted of more than 50 ESTs each, which represented the most abundant transcripts and encoded known proteins. They constituted $0.73 \%$ of the total clusters (25 of 3416 clusters) including $26.16 \%$ of total ESTs (2275 of 8696 ESTs). Interestingly, half of the most abundant transcripts were previously reported metalloproteinase from venom gland of D. acutus [11], indicating high prevalence. Of these 25 clusters, eight clusters are known genes that belong to housekeeping genes and two are toxin secretion related genes (Table 1).

(2) Thirty-nine clusters consisted of 20-49 ESTs each and represented $1.14 \%$ of the total clusters (39 of 3416 clusters) and $13.26 \%$ of the total ESTs (1153 of 8696 ESTs). Of 39 clusters, 13 encoded non-toxin functional proteins,

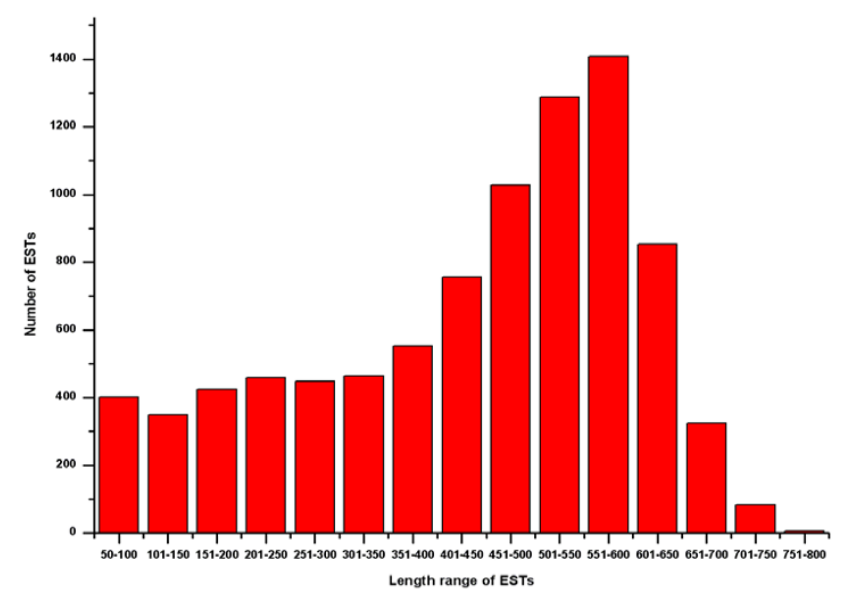

\section{Figure I}

The distribution of the reads length of the ESTs. A total of 8696 ESTs were generated in the present study. Abscissa (50 bp) is the length of sequences, while the $Y$-coordinate is the number of ESTs. A large fraction of ESTs is between 350 and 650 bp. 


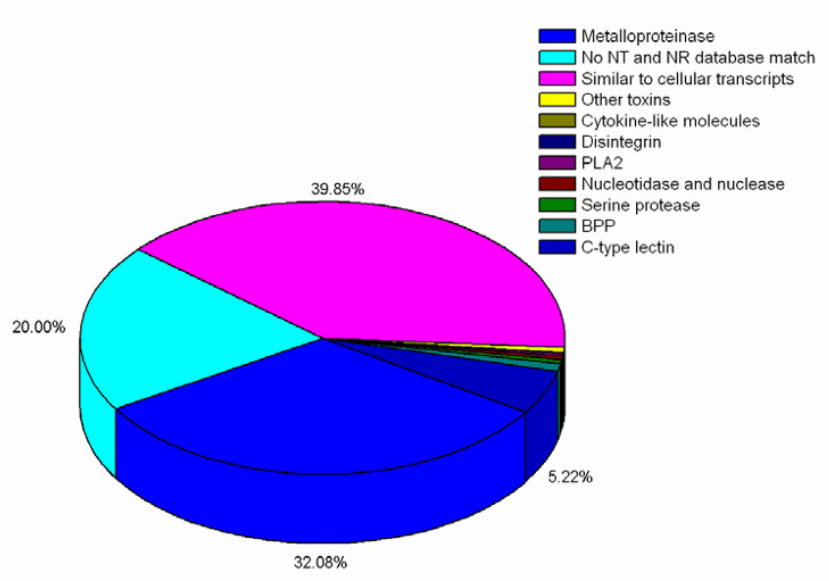

Figure 2

Distribution of ESTs from D. acutus venomous gland according to their cDNA products. The percentage of ESTs classification more than $1.00 \%$ is indicated.

such as myosin, NADH dehydrogenase subunit, cytochrome oxidase subunit and calmodulin protein. They are the second most abundant mRNA transcripts in the venom gland of $D$. acutus.

(3) Seventy-five clusters contained 10-19 ESTs each, and represented $2.20 \%$ of the total clusters (75 of 3416 clusters) and $11.83 \%$ of ESTs (1029 of 8696 ESTs), of which 17 clusters encoded the genes for troponin, ATPase subunits, retrotransposable-like elements and elongation factor 1-alpha, etc. They are considered medium-sized clusters with relatively low prevalence.

(4) The low abundant 445 clusters consisted of 2-9 ESTs each and constituted $13.03 \%$ of the total clusters (445 of 3416 clusters) and $13.69 \%$ of the total ESTs (1425 of 8696 ESTs). They included many toxin coding genes, cellular functional transcripts and partial unknown protein e.g., jerdonitin, proline dehydrogenase and hypothetical proteins.

(5) There are 2832 unique ESTs representing $82.9 \%$ of the total clusters (2832 of 3416 clusters) and $32.57 \%$ of ESTs (2832 of 8696 ESTs). The occurrence rate of these clusters is only once in current sequenced ESTs. They included cytokine-like molecule, zinc finger proteins, transport proteins and transcripts without hits to GenBank nonredundant proteins (nr) and nucleic acids databases (nt). The distribution of these cluster sizes are shown in Figure 3.

The cDNA library constructed is a non-normalized primary library without amplification, so the clone abundance or the cluster size presents the relative mRNA

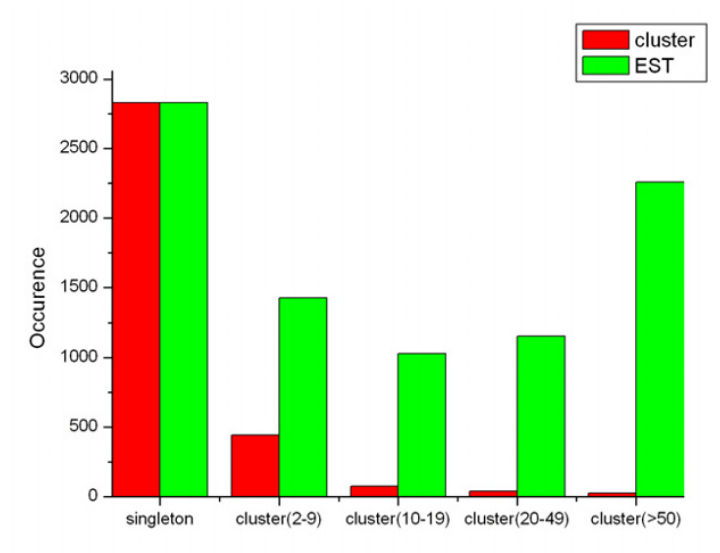

Figure 3

Prevalence distribution of the cluster size. The initial 8696 ESTs were grouped into 3416 clusters, consisting of 25 clusters (2257 of 8696 ESTs) that compromised of more than 50 ESTs each cluster, 39 clusters contained 20 49 ESTs (I I53 of 8696 ESTs), 75 clusters contained 10 19 ESTs (I029 of 8696 ESTs), 445 clusters comprised 2 9 ESTseach cluster (I 425 of 8696 ESTs), and 2832 unique sequence.

population [12]. About one-third of the total clones are singletons, and approximately one-fourth of the ESTs fit in clusters that are comprised of more than 50 ESTs, representing the complexity and specificity of the transcript population of the venom gland of D. acutus.

\section{ESTs relevant to protein processing}

A homologue of Bothrops insularis calglandulin EF-hand protein family is identified at high abundance (81 ESTs) in current library (Table 1). Calglandulin EF-hand protein as a venom gland specific gene has been reported [13]. It has several conserved $\mathrm{Ca}^{2+}$ motifs and is expressed exclusively in snake venom glands from many species, but not secreted to the venom. This protein family functions in the process of exporting toxins out of the cell and into the venom [14], implying that it plays a fundamental role in toxin secretion process [15]. In this library, three EF-hand protein families were found, of which two showed high identity with Bothrops insularis EF-hand protein family and another showed homology only with Mus musculus calmodulin. The diversity of EF-hand proteins in the venom gland of $D$. acutus may suggest the complexity of toxin secretion activity. The other high expression gene encodes the protein disulfide isomerase (PDI), which was represented by 58 ESTs in the library. The PDI from D. acutus showed $77.9 \%$ of identity with Gallus gallus PDI. The PDI is a redox protein responsible for disulfide bond assembly in the endoplasmatic reticulum. We also found a significant frequency difference of cysteine residues between toxin protein and cellular functional proteins (data not 
Table I: Identification of the high abundance cluster ( $\geq 10$ reads) of cellular functional transcripts

\begin{tabular}{|c|c|c|c|}
\hline Clustera & reads $^{b}$ & E-value & Annotationc \\
\hline uni857।478 & 217 & 0 & Creatine kinase [Zaocys dhumnades] \\
\hline uni33563240 & 190 & 0 & Actin, alpha I, skeletal muscle [Mus musculus] \\
\hline uni5359759 & 124 & $1.00 \mathrm{E}-65$ & Fast troponin $\mathrm{T}$ isoform [Coturnix japonica] \\
\hline uni2350666l & 81 & I.00E-78 & Calglandulin EF-hand protein [Bothrops insularis] \\
\hline uni6978487 & 79 & 0 & Aldolase A [Rattus norvegicus] \\
\hline uni64277 & 67 & $1.00 \mathrm{E}-170$ & Calsequestrin [Rana esculenta] \\
\hline unil3II09 & 65 & $5.00 \mathrm{E}-5 \mathrm{I}$ & Parvalbumin beta \\
\hline uni3582। 24 & 59 & 0 & Cytochrome oxidase subunit I [Dinodon semicarinatus] \\
\hline uni21703694 & 58 & 0 & Protein disulfide isomerase [Gallus gallus] \\
\hline uni45382253 & 53 & 7.00E-79 & Troponin I [Gallus gallus] \\
\hline uni23480590 & 48 & $2.00 \mathrm{E}-10$ & Hypothetical protein [Plasmodium yoelii yoelii] \\
\hline uni6708502 & 47 & 0 & Superfast myosin heavy chain [Felis catus] \\
\hline unil 8378003 & 40 & $1.00 \mathrm{E}-95$ & NADH dehydrogenase subunit I [Homoroselaps lacteus] \\
\hline uni50845428 & 29 & 8.00E-76 & Atrial/embryonic alkali myosin light chain [Homo sapiens] \\
\hline unil 2004999 & 29 & $2.00 \mathrm{E}-42$ & Fast skeletal muscle troponin T isoform 2-el6 [Mitu tomentosa] \\
\hline uni3582 128 & 28 & $1.00 \mathrm{E}-101$ & Cytochrome oxidase subunit III [Dinodon semicarinatus] \\
\hline unil 36089 & 28 & $1.00 \mathrm{E}-126$ & Tropomyosin beta chain (Tropomyosin 2) (Beta-tropomyosin) \\
\hline unil 5011037 & 24 & $1.00 \mathrm{E}-137$ & cytochrome b [Crotalus horridus atricaudatus] \\
\hline uni505। 3993 & 22 & $2.00 \mathrm{E}-74$ & Chain B, Crystal Structure Of The Sr Ca2+-Atpase With Bound Amppcp \\
\hline unil 04550 & 21 & 0 & Ca2+-transporting ATPase \\
\hline uni4604896 I & 21 & $1.00 \mathrm{E}-176$ & Glyceraldehyde-3-phosphate dehydrogenase [Gallus gallus] \\
\hline unil 68777 & 20 & $4.00 \mathrm{E}-12$ & Calmodulin-like 3 [Mus musculus] \\
\hline uni3198।562 & 20 & $1.00 \mathrm{E}-136$ & Pyruvate kinase 3 [Mus musculus] \\
\hline uni52078482 & 19 & $4.00 \mathrm{E}-92$ & Myosin light chain [Oxyuranus scutellatus scutellatus] \\
\hline uni45384350 & 15 & $4.00 \mathrm{E}-25$ & $60 \mathrm{~S}$ acidic ribosomal protein $\mathrm{PI}$ [Gallus gallus] \\
\hline uni5।460955 & 15 & $8.00 \mathrm{E}-96$ & PREDICTED: similar to CAVP-target protein (CAVPT) [Homo sapiens] \\
\hline uni45384494 & 14 & $1.00 \mathrm{E}-101$ & Acidic ribosomal phosphoprotein [Gallus gallus] \\
\hline uni3582I 27 & 14 & $6.00 \mathrm{E}-59$ & ATPase subunit 6 [Dinodon semicarinatus] \\
\hline uni3582125 & 14 & $2.00 \mathrm{E}-87$ & Cytochrome oxidase subunit II [Dinodon semicarinatus] \\
\hline uni3582।31 & 14 & $1.00 \mathrm{E}-107$ & NADH dehydrogenase subunit 4 [Dinodon semicarinatus] \\
\hline uni 47212235 & 14 & $1.00 \mathrm{E}-\mathrm{I} \mid 4$ & Unnamed protein product [Tetraodon nigroviridis] \\
\hline unil19133 & 13 & 0 & Elongation factor I-alpha (EF-I-alpha) \\
\hline uni28277353 & 13 & $7.00 \mathrm{E}-32$ & Eno3-prov protein [Xenopus laevis] \\
\hline uni86460 & 13 & $6.00 \mathrm{E}-60$ & Troponin T, skeletal muscle, isoform I - chicken \\
\hline uni330866I2 & 12 & $9.00 \mathrm{E}-48$ & Aal-330 [Rattus norvegicus] \\
\hline uni33086544 & 12 & $2.00 \mathrm{E}-59$ & Ab2-057 [Rattus norvegicus] \\
\hline uni33694242 & 12 & $1.00 \mathrm{E}-100$ & Ribosomal protein LI5 [Homo sapiens] \\
\hline uni45382453 & 11 & $1.00 \mathrm{E}-107$ & Elongation factor 2 [Gallus gallus] \\
\hline uni6576738 & 10 & $6.00 \mathrm{E}-98$ & ORF2 [Platemys spixii] \\
\hline uni4538206I & 10 & $1.00 \mathrm{E}-129$ & $\begin{array}{l}\text { Triosephosphate isomerase (TIM, D-glyceraldehyde 3-phosphate ketol-isomerase) } \\
\text { [Gallus gallus] }\end{array}$ \\
\hline
\end{tabular}

a Unigenes were obtained using Phrap software and clustered manually. b Number of sequenced clones in unigene.

c The best match from BLASTX.

shown) in the venom gland of D. acutus, suggesting that the PDI plays a key role in toxin protein folding. Furthermore, heat shock proteins (HSPs) are also identified (15 ESTs) in this library including HSP20, HSP70 and HSP90. HSPs are chaperon for protein refolding and degeneration, which is possibly important to toxin proteins regeneration. There are many ribosomal proteins found in this library, which contributes to the high level of protein synthesis events. A large number of ribosomal proteins therefore are needed for the toxins synthesis [16]. Several other identified transcripts can also shed light on the physiological aspects of the venom gland secretion style. For instance, various clusters involved in transporter activity are found, e.g., ion transporters (uni4929105), nucleoside transporters (uni7320865) (see Additional File). All these suggested that the venom gland of $D$. acutus is a highly specialized active organ that plays a central role in secreting toxins and polypeptides with powerful synthesis capabilities.

\section{ESTs relevant to structural components and energy supply} There are abundant structural component transcripts expressed in the venom gland of $D$. acutus, encoding actin, troponin, calsequestrin and myosin (Table 1). Interest- 
ingly, these cellular structural components from the venom gland are similar to ones from mammalian muscle tissue, which may indicate that the structure of the venom gland cavity is similar to muscle tissue and contributes to the venom gland contractile activity. Accordingly, it could be explained that creatine kinase expressed highly in the venom gland of $D$. acutus, accounting for 2.49\% (217 reads) of all ESTs (Table 1). Because creatine kinase is an important enzyme regulator of high-energy phosphate production and utilization within contractile tissues, high expression of the enzyme is adapted to energy need for gland contraction. Furthermore, abundant transcripts expressed in current library also involved in cytochrome b, cytochrome oxidase and NADH dehydrogenase, which are also needed to meet energy needs for toxin protein synthesis and gland contraction.

\section{Enzymes relevant to metabolism pathway}

In this library, several enzymes in metabolic pathways such as glucose metabolism and nicotinate and nicotinamide metabolism were found (Table 2). In energy and material metabolism, 22 clusters sequences were identified to play a role in glucose metabolism. We also identified that unigene uni4505467 and unigene uni41055552 code for the 5 '-nucleotidase, which suggests that $D$. acutus may possess a functional pathway for purine metabolism and nicotinate and nicotinamide metabolism in the venom gland. The 5 '-nucleotidase participates not only in purine metabolism but also in nicotinate and nicotinamide metabolism. Snake envenomation employs three well-integrated strategies: prey immobilization via hypotension, prey immobilization via paralysis, and prey digestion. Purines (adenosine, guanosine and inosine) constitute the perfect multifunctional toxins, and evidently play a central role in all three envenomation strategies of most advanced snakes [17].

\section{ESTs relevant to other function}

Surprisingly, 18 clusters (21 ESTs) encoding for reverse transcriptase were found in current library. They are similar to reverse transcriptase from teleost LINE family SW1 [18]. At the same time, we identified retrotransposablelike elements in this library (16 ESTs), most of them similar to ORF2 protein from a Platemys spixii retrotransposon CR1 [19]. So we could expect an intact retrotransposable structure in the D. acutus genome. This specific retrotransposable element in the $D$. acutus genome may be adapted to environmental diversity and prey need.

So far, we still have not determined the complete functional categories of genes expressed in the venom gland of snake. To give an overview of the major cellular roles, the number of partial mRNA transcripts represented in each category is listed (Additional file) based on molecular function of Gene Ontology [20]. A major proportion (38 clusters) represent transcripts involved in the binding category, corresponding to $34.86 \%$ of genes of molecular function and $1.33 \%$ of total unigenes, respectively. Based on Gene Ontology function classification, 824 clusters (3144 ESTs) are assigned into the organizing principle of molecular function and 1719 clusters (5472 ESTs) of biological process (Figure 4). However, such an analysis only gave a hint of what the function might be and, in many cases, extensive biochemical and biological work is necessary to unambiguously identify a gene and its function [21]. We have presented here an initial analysis of those relevant to physiological cellular proteins.

\section{ESTs identifying no significant matches to known genes}

There are 1553 clusters (54.40\%) without significant homology to any known genes in GenBank. According to sequences discarding criteria (less than $50 \mathrm{bp}$ ), we could exclude the possibility that too short sequences lead to no hits. The high abundant novel sequences represent a large number of unidentified genes, suggesting the complexity and diversity of genes expressed in the venom gland of $D$. acutus. In addition, among those clusters without significant matches to known genes, 11 clusters have matches with the dbEST database and 344 clusters with the hmmpfam database. Some of those clusters, such as unigene rfstca0_000120.y1.scf showed a putative toxin-related motif region of disintegrin, and unigene rfstda0_001953.y1.scf for Conotoxin I-superfamily, indicated new toxins among those sequences. The high abundance of these sequences might correspond to the unknown toxin genes stored in the venom gland of $D$. acutus. Further study of these novel genes expressed in this specialized organ could disclose the mechanism of toxin secretion and the evolution of the snake venom gland.

\section{Comparative analysis with other snakes venom glands}

Although several cDNA libraries from the venomous gland of a few snakes have been reported and characterized $[10,15,22]$, analysis of transcripts from these cDNA libraries seldom involve cellular functions as the main attention was focused on the toxin components. Many components of toxin in the venom gland have been identified [11], but nerve growth factor (NGF) has not been identified in this library. In contrast to previous reports $[10,15]$, we postulate an alternative possibility for not identifying NGF in this library: one is that the NGF might express under the specifically physiological conditions, such as in milking venom gland of $D$. acutus; another is that NGF is not a necessary component of toxin of $D$. acutus. Furthermore, a lot of clusters that may be involved in many physiological process of venom gland remained to be deciphered. It is significant to study the gene expression of venom gland of snake on cellular structure and functional aspects, which will improve the study of some physiological process such as organogenesis, cell differen- 
Table 2: Proteins from venom gland of $D$. acutus predicted to play a role in physiological metabolism pathway

\begin{tabular}{|c|c|c|}
\hline Unigene & EC number & Enzyme name and synonyms \\
\hline \multicolumn{3}{|c|}{ Glucose metabolism } \\
\hline Unil351884 & I.I.I.I & Alcohol dehydrogenase, zinc-containing \\
\hline Uni34555782 & I.I.I.27 & L-lactate dehydrogenase \\
\hline Unil 7369829 & I.I.1.27 & L-lactate dehydrogenase \\
\hline Uni4604896I & 1.2.1.12 & Glyceraldehyde 3-phosphate dehydrogenase \\
\hline Uni5075448I & I.2.4.1 & $\begin{array}{l}\text { Pyruvate dehydrogenase complex, EI } \\
\text { component, alpha subunit }\end{array}$ \\
\hline Uni50760204 & 2.3.1.12 & $\begin{array}{l}\text { Acetoin dehydrogenase complex, E2 } \\
\text { component, dihydrolipoamide } \\
\text { acetyltransferase, putative }\end{array}$ \\
\hline Uni45383682 & 2.7.1.11 & 6-phosphofructokinase \\
\hline Uni206205 & 2.7.1.40 & Pyruvate kinase \\
\hline Uni31981562 & 2.7.1.40 & Pyruvate kinase \\
\hline Uni45384486 & 2.7.2.3 & Phosphoglycerate kinase \\
\hline Uni51950293 & 3.1 .3 .13 & Unknown \\
\hline Uni50593010 & 3.1 .3 .13 & Unknown \\
\hline Uni34867424 & 3.6.1.7 & Putative acylphosphatase \\
\hline Uni6978487 & 4.1.2.13 & Fructose-bisphosphate aldolase \\
\hline Unil 8307578 & 4.1.2.13 & Fructose-bisphosphate aldolase \\
\hline Uni28277353 & 4.2.1.1I & Enolase \\
\hline Uni2006763I & 5.3.1.9 & Glucose-6-phosphate isomerase \\
\hline Uni51950293 & 5.4.2.1 & $\begin{array}{l}\text { Similar to 2,3-diphosphoglycerate-dependent } \\
\text { phosphoglycerate mutase }\end{array}$ \\
\hline Uni50593010 & 5.4.2.1 & $\begin{array}{l}\text { Similar to 2,3-diphosphoglycerate-dependent } \\
\text { phosphoglycerate mutase }\end{array}$ \\
\hline Uni5 I950293 & 5.4.2.4 & Unknown \\
\hline Uni50593010 & 5.4.2.4 & Unknown \\
\hline Uni47224301 & 6.2.1.1 & Acetyl-CoA synthetase \\
\hline \multicolumn{3}{|c|}{ Purine metabolism } \\
\hline Uni4I393093 & I.I.I.205 & Similar to IMP dehydrogenase \\
\hline Uni53। 29754 & I.I.I.205 & Similar to IMP dehydrogenase \\
\hline Uni5I5I3483 & 2.4.2.7 & Adenine phosphoribosyltransferase \\
\hline Uni601873 & 2.4.2.8 & Hypoxanthine phosphoribosyltransferase \\
\hline Uni206205 & 2.7.1.40 & Pyruvate kinase \\
\hline Uni31981562 & 2.7.1.40 & Pyruvate kinase \\
\hline Uni50745642 & 2.7 .6 .1 & Ribose-phosphate pyrophosphokinase \\
\hline Uni28278826 & 2.7.7.6 & DNA-directed RNA polymerase, beta subunit \\
\hline Uni34873368 & 2.7.7.6 & DNA-directed RNA polymerase, beta subunit \\
\hline Unil 8032797 & 2.7 .7 .6 & DNA-directed RNA polymerase, beta subunit \\
\hline Uni5739210 & 2.7.7.7 & DNA polymerase III, epsilon subunit \\
\hline Uni4 1055552 & 3.1 .3 .5 & 5'-nucleotidase \\
\hline Uni4505467 & 3.1.3.5 & 5'-nucleotidase \\
\hline Uni50731966 & 4.6.1.1 & $\begin{array}{l}\text { Putative adenylate/guanylate cyclase } \\
\text { transmembrane protein }\end{array}$ \\
\hline \multicolumn{3}{|c|}{ Nicotinate and nicotinamide metabolism } \\
\hline Unil 3489054 & 2.7.1.- & Probable serine/threonine-protein kinase \\
\hline Uni450783। & 2.7.1.- & Probable serine/threonine-protein kinase \\
\hline Uni45384254 & 2.7.1.- & Probable serine/threonine-protein kinase \\
\hline Uni50761892 & 2.7.1.- & Probable serine/threonine-protein kinase \\
\hline Uni50752935 & 2.7.1.- & Probable serine/threonine-protein kinase \\
\hline Uni7710088 & 2.7.1.- & Probable serine/threonine-protein kinase \\
\hline Uni4 1055552 & 3.1.3.5 & 5'-nucleotidase \\
\hline Uni4505467 & 3.1.3.5 & 5'-nucleotidase \\
\hline Uni34852937 & 3.5.1.- & UDP-3-0-acyl $\mathrm{N}$-acetylglucosamine deacetylase \\
\hline
\end{tabular}



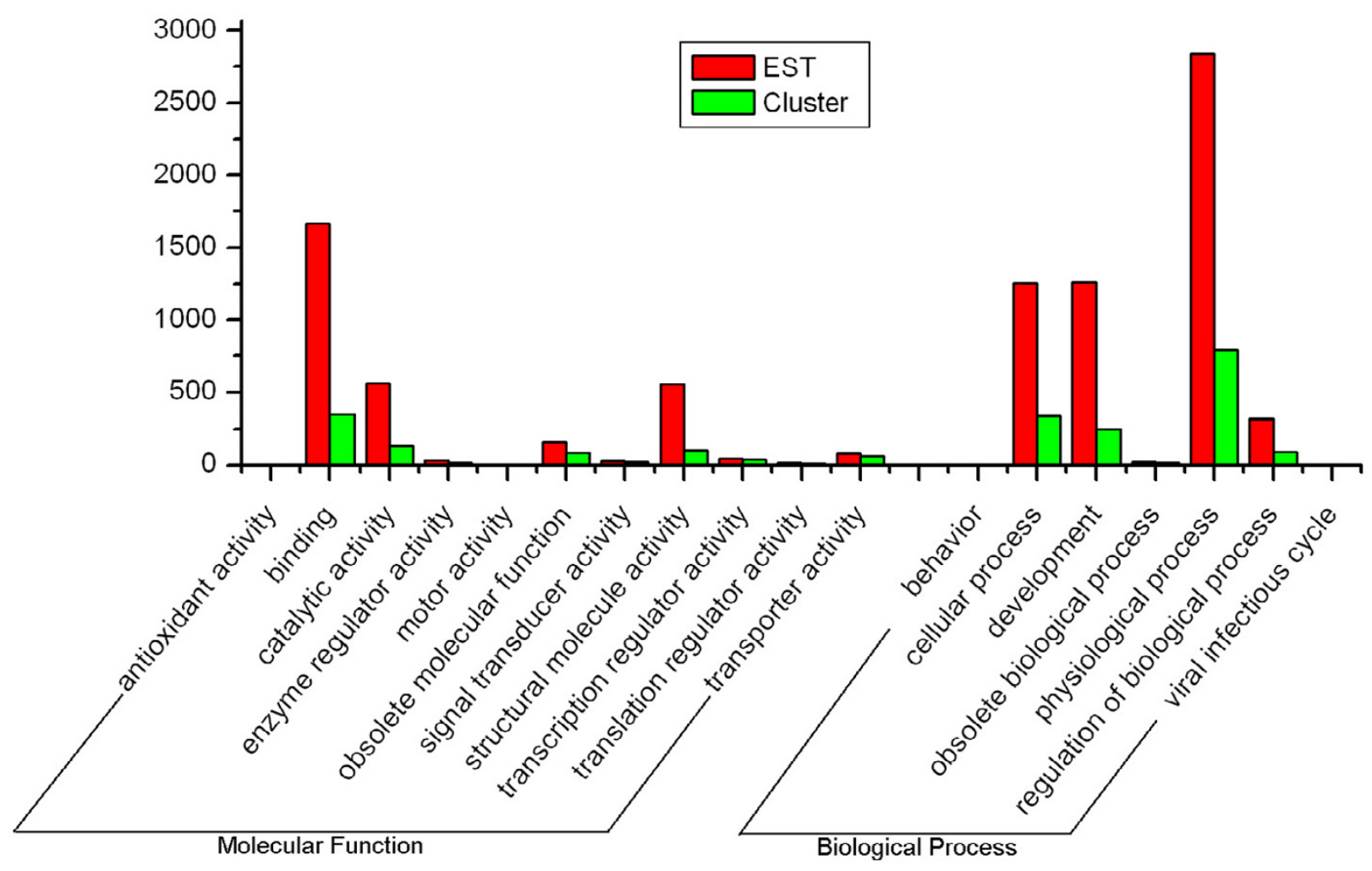

Figure 4

Functional classification of transcripts in the venom gland of $D$. acutus according to molecular function and biological process based on Gene Ontology. The vertical axis shows the number of EST or cluster.

tiation and protein synthesis. Alternatively, some secreted membrane proteins may represent antigens of potential importance to immune control.

\section{Phylogenetic analysis of toxin related genes in D. acutus} Snake venom glands evolved once, at the base of the colubroid radiation, $60-80$ million years ago, with extensive subsequent "evolutionary tinkering" $[23,24]$. The advanced snakes (superfamily Colubroidea) make up $>80 \%$ of about 2900 species of snake currently described, and contain all of the known venomous forms [1]. Generally in this library, toxin clusters match sequences from snake sources in database while the cellular functional transcripts are identified by its similarity to model organisms such as Gallus gallus, Homo sapiens and Rattus norvegicus. Of note, although those transcripts of toxin are always phylogenetically closer from another snake, the average of similarities over toxins and cellular functional transcripts has no obvious difference. From the view of evolution, we postulated that the toxins tend to diverge due to natural selective pressure to adapt to different environmental conditions (mainly distinct preys). Whereas, most of the cellular components showing similarities with mammalian proteins, although they are usually phylogenetically distant, correspond to proteins of conserved functions among vertebrates and thus show higher homology [15].

The origin and evolution of many toxin gene families in the advanced snakes have been researched extensively $[1,24,25]$. Among these toxin gene families, most were recruited into the advanced snakes before the split of elapids and viperids. However, the phylogenetic analysis of a few other toxin gene families, such as phospholipase A2 and natriuretic toxins provide a clear evidence of an independent recruitment event. Because of limited toxin gene sequences in public databases, the origin and evolution of a number of toxin gene families remain unknown. In this report, we analyzed the phylogeny of the group III snake 
venom metalloproteinase and serine protease. The group III snake venom metalloproteinase consists of a proprotein, a metalloproteinase, a disintegrin and a cysteine-rich domain. It inhibits the integrin receptor selectively. Figure 5 described the phylogenetic analysis of this group III metalloproteinase in Colubroid. The phylogenetic model of this metalloproteinase is similar to CRISP protein [24], which was recruited into the advanced snakes before the split of elapids and viperids. That is the advanced snake acquired the group III metalloproteinase genes by an early single recruitment event. Subsequently, this metalloproteinase family evolved independently in elapids and viperids. We also analyzed the phylogeny of the serine proteases in suborder Colubroidea and similar results were shown (Figure 6). But serine protease genes have not been identified in elapids, which suggested the gene loss events in the evolutionary process or insufficient sequences information of elapids.

\section{Conclusion}

This study identified and characterized the cellular functional transcripts in the venom gland of $D$. acutus extensively. Our aim is to develop a catalog of genes transcribed in this snake venom gland. We hoped to discover as many toxin and cellular coding genes as possible by constructing five fraction sub-libraries. The venom gland of D. acutus expresses many protein-coding sequences that are too divergent from those at present in GenBank to provide identification. Moreover, we found as many unidentified protein-coding sequences as identified ones. The prevalence distribution also provides a reasonable estimation of the actual frequency of these sequences in the venom gland of D. acutus. Furthermore, we have described a number of recognized molecules previously not known to be expressed in the venom gland of D. acutus, i.e., dehydrogenase and calglandulin EF-hand protein families. Though it is of relatively small size, analysis of this set of ESTs has yielded several kinds of useful information pertaining to the unmilked venom gland of $D$. acutus. It is, therefore, likely that the generation of more sequence data will result in the identification of novel $D$. acutus genes. In addition, many ESTs do not have significant database matches and these open up new avenues of exploration.

This report successfully provides evidence about the function of snake venom gland and a source of reptilian sequences. Gene cataloguing and profiling of the venom gland of $D$. acutus is an essential requisite to provide molecular reagents for functional genomic studies needed for elucidating mechanisms of action of toxins and surveying physiological events taking place in the very specialized secretory tissues. So this study provides a first global view of the genetic programs for the venom gland of $D$. acutus described so far and an insight into molecular mechanism of toxin secreting in the very specialized organ.

\section{Materials and methods Library construction}

A non-normalized cDNA library was generated from the venom gland of $D$. acutus. The $D$. acutus was captured from Wuyi Mountain, Fujian Province, China. After killing the snake by cutting the head, the venom glands were recovered. All tissues were immediately frozen in liquid nitrogen. The total RNA was isolated with Trizol Reagent (Invitrogen) and the mRNA was purified with Oligotex mRNA Kits (Qiagen). The cDNA synthesized with Superscipt II-RT (Invitrogen) and DNA polymerase I (Promega), was flanked by EcoR I adaptor (Stratagene) and treated by Xho I (Stratagene). The double strand cDNA was extracted by electrophoresis separation with five fractions (<0.25 kb, 0.25 0.5 kb, 0.5 1 kb, 1 2 kb and $>2 \mathrm{~kb}$ ) and then cloned into EcoR I and Xho I digested pBluescript II vector. The plasmid was transformed into E. coli (DH10B) to amplify the cDNA.

\section{EST sequencing, data processing and bioinformatics analysis}

The cDNA clones were picked randomly. Plasmids were isolated according to a standard alkaline lysis protocol, and sequenced with MegaBACE 1000 (Amersham Pharmacia). Base-Calling was performed with PHRED [26], the cutoff Phred score was 20 [27]. Original sequences were generated by removing vector and E. coli DNA sequences using Cross-match [26]. High-quality ESTs were assembled into contigs using Phrap software [26]. Default settings were used except 40 bp minimum overlap and $99 \%$ identity. To assign annotation to the assembled ESTs (clusters), these sequences were searched against the $\mathrm{nr}(\mathrm{E}$ values < 1e-5) and nt (E values < 1e-10) for homology comparison using BLASTX and BLASTN [28]. Then those clusters without significant matches to known genes were compared to the dbEST database by BLAST and pfam database by hmmpfam [29]. All assembled sequences having the same annotation were further clustered into a unique gene. Based on Gene Ontology classification, we constructed a gene expression profile of the venom gland of $D$. acutus. In order to comprehend the role of those transcripts played in the biochemical process, we assigned enzyme functions and enzyme commission numbers to reconstruct anabolic and catabolic pathways through linkage to biochemical pathways in the KEGG database [30]. The phylogenetic analysis of toxin protein families was conducted by MEGA 3.1 [31].

\section{Authors' contributions}

$\mathrm{ZB}$, LQ participated in the design of study, carried out the experiments and the comparative analysis, and drafted the manuscript. YW participated in the design of study. ZX, LY 


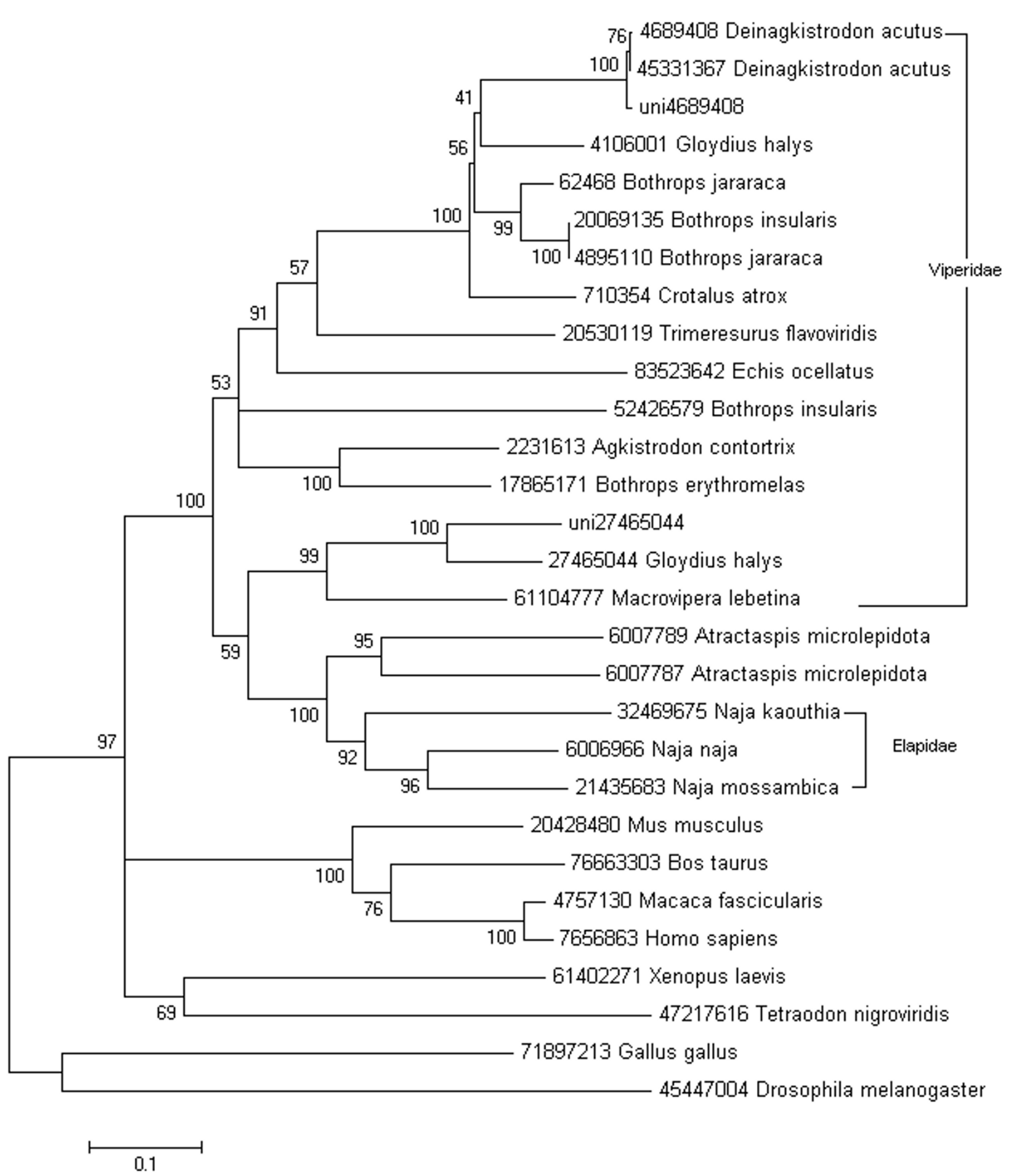

\section{Figure 5}

Molecular phylogenetic analysis of the group III metalloprotease using Neighbor-joining method. To minimize confusion, all proteins sequences from database are referred to their NCBI accession numbers. The sequences from the current library are indicated with prefix "uni". 


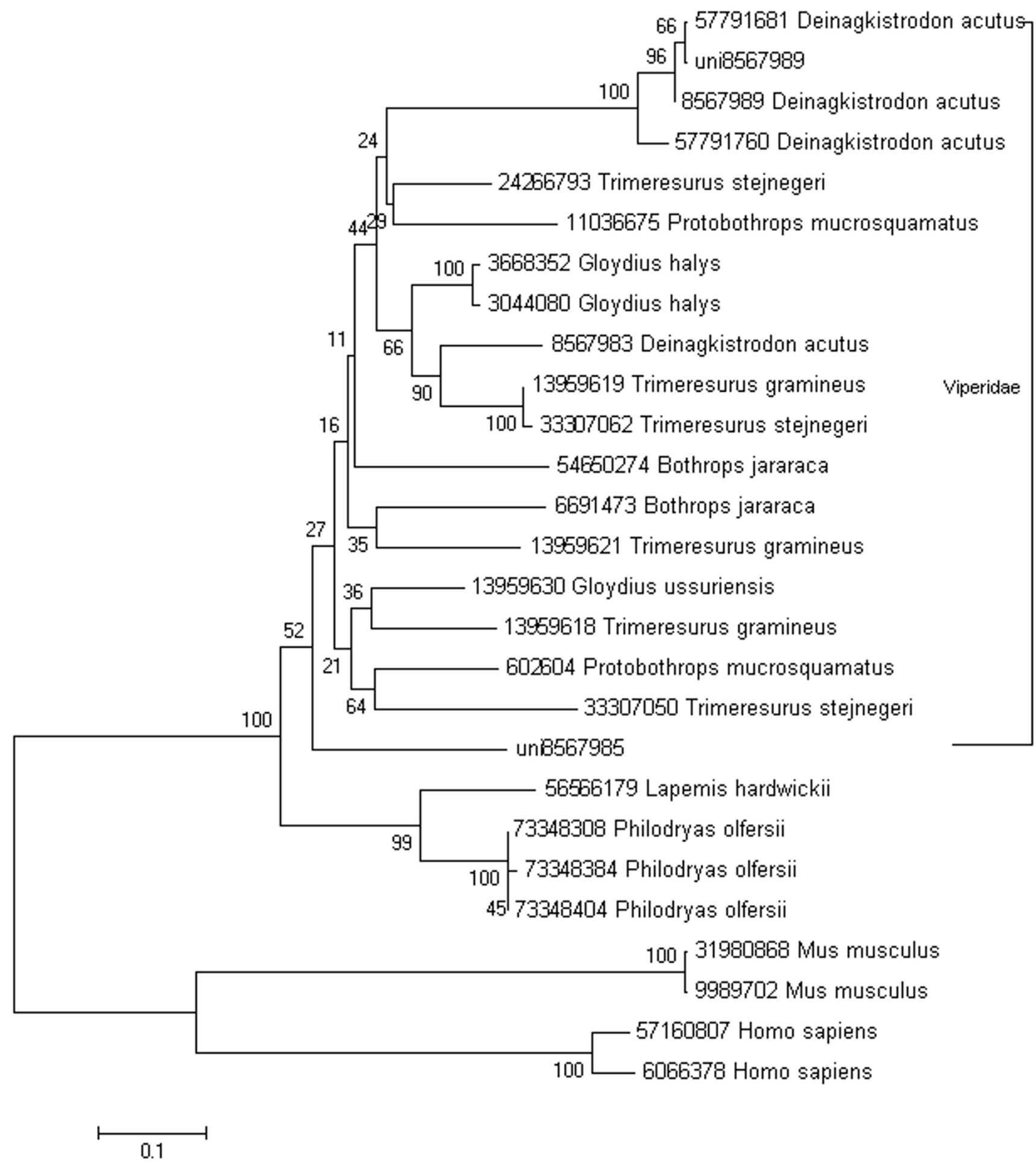

Figure 6

Molecular phylogenetic analysis of the serine proteinase using Neighbor-joining method. To minimize confusion, all proteins sequences from database are referred to their $\mathrm{NCBI}$ accession numbers. The sequences from the current library are indicated with prefix "uni". 
participated in the design of study and the data analysis. HY, QP, SX participated in the data analysis. YJ, HS, YG conceived the study and participated in coordinate and help to draft the manuscript. All authors have read and approved the paper.

\section{Acknowledgements}

We thank Ph.D. Wang Jian and Ph.D. Siqi Liu (Beijing Institute of Genomics, Chinese Academy of Sciences, Beijing, China) for technical support and helpful discussion. We also appreciate Ph.D. Mengfeng Li (Zhong-Shan Medical College, Sun Yat-sen University, Guangzhou, China) for his critical comments. We are also grateful to Dr. Ivo M.B. Francischetti (NIH, USA) and Dr. Inácio L.M.junqueira de Azevedo (Universidade de Sao Paulo, Sao Paulo, Brazil) for reference data support and helpful suggestions. We are also in debt to the editors and anonymous reviewers for patiently giving us advice for revising the manuscripts. This work was supported by a key grant of Science and Technology of Guangdong Province, China (2003A 10905), and the CAS Hundred Talents Program (to Jun Yu).

\section{References}

I. Fry BG: From genome to "venome": molecular origin and evolution of the snake venom proteome inferred from phylogenetic analysis of toxin sequences and related body proteins. Genome Res 2005, 15:403-420.

2. Ohno M, Chijiwa T, Oda-Ueda N, Ogawa T, Hattori S: Molecular evolution of myotoxic phospholipases A2 from snake venom. Toxicon 2003, 42:84I-854.

3. Guo LY, Pang S, Ruan QQ, Zhou YC: Isolation and Characterization of a Neurotrophic Factor-like Substance from Venom of Agkistrodon acutus. Sheng Wu Hua Xue Yu Sheng Wu Wu Li Xue Bao (Shanghai) 1999, 31:2II-214.

4. Zang J, Zhu Z, Yu Y, Teng M, Niu L, Huang Q, Liu Q, Hao Q: Purification, partial characterization and crystallization of acucetin, a protein containing both disintegrin-like and cysteinerich domains released by auto-proteolysis of a P-III-type metalloproteinase AaH-IV from Agkistrodon acutus venom. Acta Crystallogr D Biol Crystallogr 2003, 59:23I 0-23I2.

5. Zha XD, Liu J, Xu KS: cDNA cloning, sequence analysis, and recombinant expression of akitonin beta, a C-type lectin-like protein from Agkistrodon acutus. Acta Pharmacol Sin 2004, 25:372-377.

6. Zhu Z, Liang Z, Zhang T, Zhu Z, Xu W, Teng M, Niu L: Crystal structures and amidolytic activities of two glycosylated snake venom serine proteinases. I Biol Chem 2005, 280: 10524-10529.

7. Adams MD, Kelley JM, Gocayne JD, Dubnick M, Polymeropoulos MH, Xiao H, Merril CR, Wu A, Olde B, Moreno RF, et al.: Complementary DNA sequencing: expressed sequence tags and human genome project. Science 1991, 252:165I-1656.

8. Takasuga A, Hirotsune S, Itoh R, Jitohzono A, Suzuki H, Aso H, Sugimoto $Y$ : Establishment of a high throughput EST sequencing system using poly(A) tail-removed cDNA libraries and determination of $\mathbf{3 6 , 0 0 0}$ bovine ESTs. Nucleic Acids Res 200I, 29:EI08.

9. Kore-eda S, Cushman MA, Akselrod I, Bufford D, Fredrickson M, Clark E, Cushman JC: Transcript profiling of salinity stress responses by large-scale expressed sequence tag analysis in Mesembryanthemum crystallinum. Gene 2004, 341:83-92.

10. Kashima S, Roberto PG, Soares AM, Astolfi-Filho S, Pereira JO, Giuliati S, Faria M Jr, Xavier MA, Fontes MR, Giglio JR, Franca SC: Analysis of Bothrops jararacussu venomous gland transcriptome focusing on structural and functional aspects: I - gene expression profile of highly expressed phospholipases A2. Biochimie 2004, 86:2II-219.

II. Liu QH, Zhang XW, Yin W, Li C], Huang YJ, Qing PX, Su XW, Hu $\mathrm{SN}$, Yan GM: A catalog for transcripts in the venom gland of the Agkistrodon acutus: Identification of the toxins potentially involved in coagulopathy. Biochemical and Biophysical Research Communications 2006, 341:522-531.

12. Mou CY, Zhang SC, Lin JH, Yang WL, Wu WY, Wei JW, Wu XK, Du JC, Fu ZY, Ye LT, Lu Y, Xie XJ, Wang YL, Xu AL: EST analysis of
mRNAs expressed in neurula of Chinese amphioxus. Biochem Biophys Res Commun 2002, 299:74-84.

13. Pierre LS, Woods R, Earl S, Masci PP, Lavin MF: Identification and analysis of venom gland-specific genes from the coastal taipan (Oxyuranus scutellatus) and related species. Cell Mol Life Sci 2005, 62:2679-2693.

14. Junqueira-de-Azevedo Ide L, Pertinhez T, Spisni A, Carreno FR, Farah CS, Ho PL: Cloning and expression of calglandulin, anew EFhand protein from the venom glands of Bothropsinsularis snake in E. coli. Biochim Biophys Acta 2003, 1648:90-98.

15. Junqueira-de-Azevedo Ide L, Ho PL: A survey of geneexpression and diversity in the venom glands of the pitviper snake Bothrops insularis through the generation of expressed sequence tags (ESTs). Gene 2002, 299:279-291.

16. Majumdar AP: Protein synthesis by gastric mucosal ribosomes during development in rats. J Pediatr Gastroenterol Nutr 1984, 3:123-127.

17. Aird SD: Ophidian envenomation strategies and the role of purines. Toxicon 2002, 40:335-393.

18. Duvernell DD, Turner BJ: Swimmer I, a newlow-copy-number LINE family in teleost genomes with sequence similarity to mammalian LI. Mol Biol Evol 1998, 15:179I-1793.

19. Kajikawa M, Ohshima K, Okada N: Determination of the entire sequence of turtle CRI: the first open reading frame of the turtle CRI element encodes a protein with a novel zinc finger motif. Mol Biol Evol 1997, 14:1206-1217.

20. Ashburner M, Ball CA, Blake JA, Botstein D, Butler H, Cherry JM, Davis AP, Dolinski K, Dwight SS, Eppig JT, Harris MA, Hill DP, IsselTarver L, Kasarskis A, Lewis S, Matese JC, Richardson JE, Ringwald M, Rubin GM, Sherlock G: Gene ontology: tool for the unification ofbiology. The Gene Ontology Consortium. Nat Genet 2000, 25:25-29.

21. Delseny M, Cooke R, Raynal M, Grellet F: The Arabidopsis thaliana CDNA sequencing projects. FEBS Lett 1997, 403:22I-224.

22. Francischetti IM, My-Pham V, Harrison J, Garfield MK, Ribeiro JM: Bitis gabonica (Gaboon viper) snake venom gland: toward a catalog for the full-length transcripts (cDNA) and proteins. Gene 2004, 337:55-69.

23. Vidal N, Hedges SB: Higher-level relationships ofcaenophidian snakes inferred from four nuclear and mitochondrial genes. CR Biol 2002, 325:987-995

24. Fry BG, Wuster W: Assembling an arsenal: origin and evolution of the snake venom proteome inferred from phylogenetic analysis of toxin sequences. Mol Biol Evol 2004, $21: 870-883$.

25. Fry BG, Wuster W, Kini RM, Brusic V, Khan A, Venkataraman D Rooney AP: Molecular evolution and phylogeny of elapid snake venom three-finger toxins. J Mol Evol 2003, 57: I I0-129.

26. Laboratory of PHIL GREEN Site [http://www.phrap.org/]

27. Ewing B, Hillier L, Wendl MC, Green P: Base-calling of automated sequencer traces using phred. I. Accuracy assessment. Genome Res 1998, 8:175-185.

28. Altschul, Stephen F, Thomas Madden L, Alejandro Schaffer A, Jinghui Zhang, Zheng Zhang, Webb Miller, David Lipman J: Gapped BLAST and PSI-BLAST: a new generation of protein database search programs. Nucleic Acids Res 1997, 25:3389-3402.

29. Krogh A, Brown M, Mian IS, Sjolander K, Haussler D: Hidden Markov models in computational biology. Applications to protein modeling. J Mol Biol 1994, 235:I50I-I53I.

30. Kanehisa M, Goto S, Kawashima S, Okuno Y, Hattori M: The KEGG resource for deciphering the genome. Nucleic Acids Res 2004, 32:D277-280

31. Kumar S, Tamura K, Nei M: MEGA3: Integrated software for Molecular Evolutionary Genetics Analysis and sequence alignment. Brief Bioinform 2004, 5: 150-163. 